\title{
Inhibition of Drug-Metabolizing Enzyme Activity in Human Hepatic Cytochrome P450s by Bisphenol A
}

\author{
Toshiro Niwa, ${ }^{a, 1)}$ Miki Tsutsui, ${ }^{a}$ Kae Kishimoto,,${ }^{b}$ Yoshiyasu YabusaKi, ${ }^{b}$ Fumihide Ishibashi, ${ }^{a}$ and \\ Masanao KATAGIRI ${ }^{*, a}$ \\ Division of Natural Science, Osaka Kyoiku University, ${ }^{a} 4$ Asahiga-oka, Kashiwara, Osaka 582-8582, Japan and

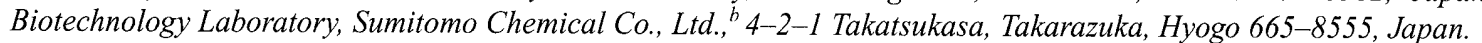 \\ Received September 20, 1999; accepted December 19, 1999
}

\begin{abstract}
Effect of bisphenol A on drug-metabolizing enzyme activities by human hepatic cytochrome P450s (CYP) was investigated. We measured aminopyrine $N$-demethylation by eleven kinds of cDNA-expressed CYPs. CYP2C19 and CYP2B6 catalyzed most efficiently the aminopyrine $N$-demethylation, followed by CYP2C8 and CYP2D6. Bisphenol A (1 mM) most efficiently inhibited aminopyrine $N$-demethylation by CYP2C8 and CYP2C19 by $82 \%$ and $85 \%$, respectively, whereas inhibition of the activities by CYP 2B6 and 2D6 was less than $40 \%$. Bisphenol A exhibited a noncompetitive-type inhibition of aminopyrine $N$-demethylase activity by CYP2C8 with $K_{\mathrm{i}}$ value of $97 \mu \mathrm{M}$. Additionally, we investigated the inhibitory effect of bisphenol A on CYP2C19-mediated $S$ mephenytoin 4-hydroxylation. Bisphenol A exhibited a mixed-type inhibition with $K_{\mathrm{i}}$ value of $113 \mu \mathrm{M}$. These results suggest that bisphenol A inhibits human hepatic CYP activities, especially CYP2C8 and CYP2C19.
\end{abstract}

Key words bisphenol A; aminopyrine $N$-demethylation; $S$-mephenytoin 4-hydroxylation; human hepatic cytochrome P450 inhibition

Bisphenol A (4,4'-isopropylidenediphenol, Fig. 1) is widely used in the chemical industry in the manufacturing of epoxy, polycarbonate, and polyester-styrene resins, and trace levels of bisphenol A leach out from polycarbonate plasticware and resins used for food packaging materials. It is remarkable that this compound seems to be an environmental estrogen-like chemical. ${ }^{2)}$ Additionally, it has been reported that exposure of bisphenol A to male rats and mice may be associated with increased cancers of the hematopoietic system and that high doses of bisphenol A cause reproductive toxicity and affect cellular development in these species. ${ }^{3)}$ However, the effect of bisphenol A in vivo is still unclear.

Cytochrome P450s (CYPs) comprise a superfamily of enzymes that catalyze the oxidation of a wide variety of xenobiotic chemicals including drugs, carcinogens, and steroids including sex hormones. ${ }^{4,5}$ Bisphenol $\mathrm{A}$ is metabolized to DNA-reactive bisphenol-O-quinone through 5-hydroxybisphenol and bisphenol semiquinone in rats, ${ }^{6,7)}$ and the formation of the DNA adducts in a microsomal activation system is markedly decreased by CYP inhibitors, ${ }^{6}$ suggesting that CYPs are closely associated with the metabolism and toxicity of bisphenol A. Additionally, Hanioka et al. ${ }^{8)}$ reported that the treatment of bisphenol $\mathrm{A}$ on male rats decreased the catalytic activities and protein levels of male-specific CYP isoforms such as CYP2C11 and CYP3A2 in rat liver microsomes. However, there are no reports on the effect of bisphenol A describing its direct human CYP-mediated drug metabolizing activity.

Recently the expression systems for human CYPs have been developed in Escherichia coli, in the yeast Saccharomyces cerevisiae, and in human Hep G2 cells, and these genetic technologies have been shown to be very useful in determining the metabolism of xenobiotics by different CYPs. ${ }^{9-12)}$ We reported that the aminopyrine $N$-demethylation process is metabolized by most of the human hepatic CYPs measured, ${ }^{13)}$ indicating that this is useful to investigate the effect of inhibitors on human hepatic CYPs. In the present study, we investigated the inhibitory effect of bisphenol

* To whom correspondence should be addressed.
A on drug-metabolizing enzyme activities by the human hepatic CYPs using the cDNA-expressed P450s.

\section{MATERIALS AND METHODS}

Materials Aminopyrine (Fig. 1) and phenobarbital were obtained from Wako Pure Chemical Industries, Ltd. Bisphenol A was purchased from Tokyo Chemical Industry Co., Ltd. S-Mephenytoin (Fig. 1) and 4-hydroxymephenytoin were obtained from Sumika Chemical Analysis Service, Ltd. All other reagents were of the highest purity commercially available.

Expression of Human CYPs and Preparation of Microsomes Expression of human hepatic CYPs in recombinant Saccharomyces cerevisiae and preparation of microsomal fractions from the yeast cells were carried out according to the methods described previously. ${ }^{12,13)}$ The contents of P450s were determined as described by Omura and Sato. ${ }^{14)}$

Assay of Aminopyrine $N$-Demethylase Activity Aminoipyrine $N$-demethylase activity was measured by the method described. ${ }^{13)}$ For standard incubation, the final reaction mixture $(0.5 \mathrm{ml})$ contained yeast microsomes $(50 \mathrm{pmol}$

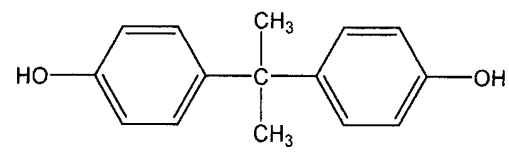

Bisphenol A
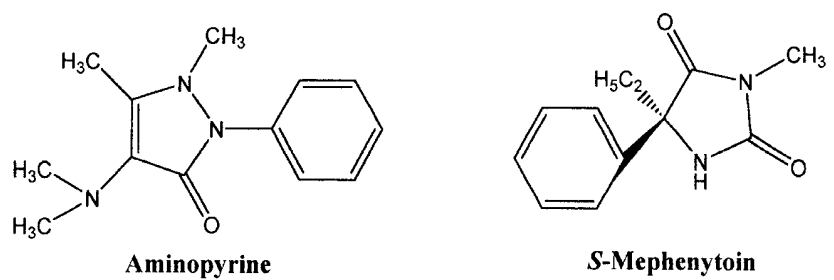

Fig. 1. Chemical Structures of Bisphenol A, Aminopyrine, and $S$ Mephenytoin

C 2000 Pharmaceutical Society of Japan 


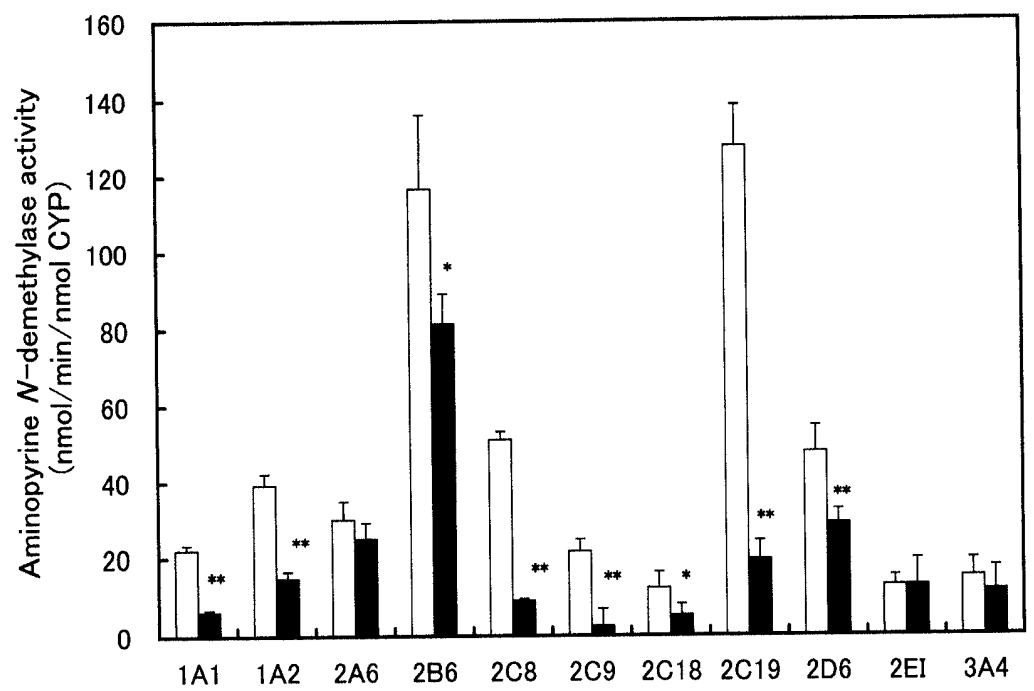

Fig. 2. Inhibitory Effect of Bisphenol A on Aminopyrine $N$-Demethylation by Eleven Kinds of Human Hepatic CYPs

Open box shows the activity in the absence of bisphenol $\mathrm{A}$ and closed box the activity in the presence of $1 \mathrm{~mm}$ bisphenol A. Results are means \pm S.E. of $3-4$ experiments. $* p<0.05, * * p<0.01$.

CYP), $2 \mathrm{~mm}$ aminopyrine, $1 \mathrm{~mm}$ NADPH, and $100 \mathrm{~mm}$ Tris$\mathrm{HCl}$ buffer $\left(\mathrm{pH} \mathrm{7.5)}\right.$. Afetr a $3 \mathrm{~min}$ preincubation at $37^{\circ} \mathrm{C}$, the reaction was started by adding NADPH. Incubation was carried out at $37^{\circ} \mathrm{C}$ for $5 \mathrm{~min}$ and the reaction was stopped by the addition of $0.25 \mathrm{ml}$ of $20 \%$ trichloroacetic acid. Aminopyrine $N$-demethylase activity was determined by estimation of formaldehyde production using the Nash reagent. ${ }^{15)}$

Assay of $S$-Mephenytoin 4-Hydroxylase Activity The incubation mixture consisted of yeast microsomes $(50 \mathrm{pmol}$ CYP), $10-100 \mu \mathrm{M} S$-mephenytoin, $1 \mathrm{~mm} \mathrm{NADPH}$, and 100 $\mathrm{mm}$ Tris- $\mathrm{HCl}$ buffer $(\mathrm{pH} \mathrm{7.5)}$ in a final volume of $0.5 \mathrm{ml}$. After a 2 min preincubation at $37^{\circ} \mathrm{C}$ with or without bisphenol $\mathrm{A}$, the reaction was started by adding NADPH. Incubation was carried out at $37^{\circ} \mathrm{C}$ for $5 \mathrm{~min}$, the reaction was terminated with $3 \mathrm{ml}$ of dichloromethane, and $50 \mu \mathrm{l}$ of $40 \mu \mathrm{m}$ phenobarbital was added as an internal standard. After dichloromethane extraction, the organic phase $(2.5 \mathrm{ml})$ was evaporated under nitrogen, the residue was dissolved in 200 $\mu \mathrm{l}$ of the HPLC mobile phase and analyzed by HPLC. The HPLC system consisted of an L-7100 pump, an L-7400 UVdetector set at $224 \mathrm{~nm}$, and a D-7500 integrator (Hitachi). A column $(4.6 \times 250 \mathrm{~mm})$ packed with Cosmosil 5 C18-AR-II (Nacalai Tesque) was used. The mobile phase consisted of $7.5 \mu \mathrm{M}$ phosphoric acid/acetonitrile $(80: 20)$, and the flow rate was $1 \mathrm{ml} / \mathrm{min}$. The calibration curve was linear for concentrations of 4-hydroxymephenytoin ranging from 0.2 to 20 $\mu \mathrm{M}$, and the coefficients of variation were less than $5.5 \%$.

Kinetic Analysis $V_{\max }$ and $K_{\mathrm{m}}$ values for aminopyrine $N$ demethylase and $S$-mephenytoin 4-hydroxylase activities were determined by fitting to Michaelis-Menten kinetics by Lineweaver-Burk plots, and inhibition constants ( $K_{\mathrm{i}}$ values) were calculated according to Dixon. ${ }^{16)}$

Statical Analysis Statical analysis was performed by Student's $t$-test.

\section{RESULTS}

The inhibitory effect of bisphenol A (1 mM) on the $N$ demethylation of aminopyrine was investigated on eleven forms of human hepatic CYPs (Fig. 2). Aminopyrine $\mathrm{N}$ demethylation was catalyzed most efficiently by CYP2C19 and CYP2B6. CYP2C8 and CYP2D6 showed $37-40 \%$ of the activity by CYP2C19. CYP1A2, 2A6, 1A1, and 2C9 could catalyze the $N$-demethylation reaction, but much lower than CYP2C19 (17-31\%), and the activities catalyzed by $\mathrm{CYP} 2 \mathrm{C} 18,3 \mathrm{~A} 4$ and $2 \mathrm{E} 1$ were less than $12 \%$ of those by CYP2C19. Bisphenol A strongly inhibited aminopyrine $\mathrm{N}$ demethylation by CYP2C 8 and CYP2C 19 by $82 \%$ and $88 \%$, respectively, while inhibition of the CYP2B6 and CYP2D6 activities was lower than those by CYP2C8 and CYP2C19 (30-39\% inhibition). Although CYP1A1, 1A2, 2C9, and $2 \mathrm{C} 18$ showed the lower aminopyrine $\mathrm{N}$-demethylase activities than CYP2C19 and CYP2B6, these activities were also inhibited $56-88 \%$ by bisphenol A, whereas inhibition of activities in CYP2A6, 2E1, and 3A4 was less than $23 \%$.

The inhibitory effect of bisphenol A on aminopyrine $\mathrm{N}$ demethylase activity by CYP2C 8 was further investigated in the concentration ranging from 15.6 to $250 \mu \mathrm{M}$ (Fig. 3). In the absence of bisphenol A, $K_{\mathrm{m}}$ values for aminopyrine $\mathrm{N}$ demethylase activity by CYP2C 8 was $3.83 \mathrm{~mm}$. In the presence of increasing bisphenol A concentrations, a concentration dependent inhibition of aminopyrine $N$-demethylation was observed characterized by a decreasing $V_{\max }$ value, whereas $K_{\mathrm{m}}$ value remained constant. This is consistent with a noncompetitive-type inhibition of aminopyrine $\mathrm{N}$-demethylation by bisphenol A, and the $K_{\mathrm{i}}$ value for bisphenol A was calculated to be $97 \mu \mathrm{m}$.

Bisphenol A inhibited most efficiently aminopyrine $N$ demethylation by $\mathrm{CYP} 2 \mathrm{C} 19$ (Fig. 2). It is reported that $S$ mephenytoin 4-hydroxylation is predominantly catalyzed by human hepatic CYP2C19. ${ }^{17)}$ Therefore, we investigated the inhibitory effect of bisphenol A on CYP2C19-mediated $S$ mephenytoin 4-hydroxylase activity. In the absence of bisphenol A, $K_{\mathrm{m}}$ and $V_{\max }$ values for $S$-mephenytoin 4-hydroxylase activity by CYP2C19 were $37.5 \mu \mathrm{M}$ and 53.2 $\mathrm{nmol} / \mathrm{min} / \mathrm{nmol} \mathrm{CYP}$, respectively, investigated in $S$-mephenytoin concentrations ranging from 10 to $100 \mu \mathrm{M}$. In the presence of increasing bisphenol A concentrations, the inhibition was characterized by a concentration-dependent increase in 
(A)

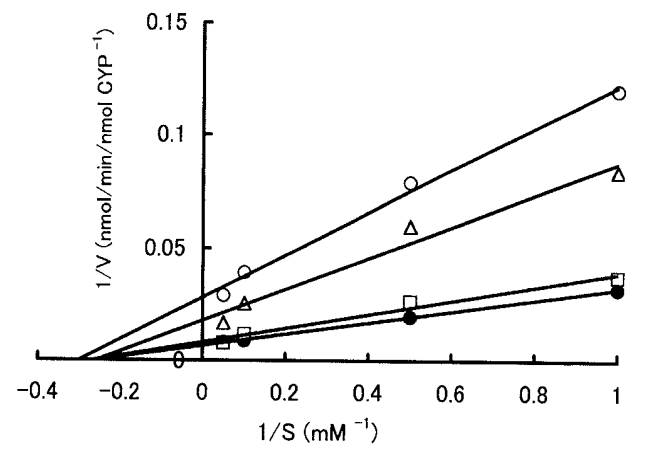

(B)

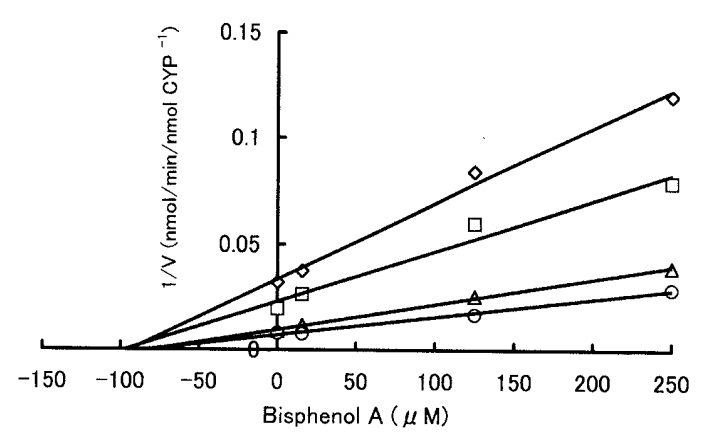

Fig. 3. Inhibitory Effect of Bisphenol A on Aminopyrine $N$-Demethylation by CYP2C8

(A) Lineweaver-Burk presentation of the inhibitory effect of bisphenol A on aminopyrine $\mathrm{N}$-demethylation by $\mathrm{CYP} 2 \mathrm{C} 8$. CYP2C 8 was incubated for $5 \mathrm{~min}$ with 1 mM NADPH and aminopyrine concentrations ranging from 1 to $20 \mu \mathrm{M}$ in the absence (๑) or the presence of $15.6 \mu \mathrm{M}(\square), 125 \mu \mathrm{M}(\triangle)$, or $250 \mu \mathrm{M}(O)$ bisphenol A. (B) Dixon plot illustrating the aminopyrine-bisphenol A interaction. The concentrations of aminoyprine were $1 \mathrm{~mm}(\diamond), 2 \mathrm{~mm}(\square), 10 \mathrm{~mm}(\triangle)$, and $20 \mathrm{~mm}(\bigcirc)$.

$K_{\mathrm{m}}$ values as well as a decrease in $V_{\max }$ values. This is consistent with a mixed-type inhibition, and the $K_{\mathrm{i}}$ value of bisphenol A was calculated to be $113 \mu \mathrm{M}$ (Fig. 4).

\section{DISCUSSION}

We investigated the effect of bisphenol A on the human CYP activities using aminopyrine as a substrate, because most of the CYPs showed the aminopyrine $N$-demethylase activity. ${ }^{13)}$ The activities by CYPs except for CYP2E1 were inhibited by bisphenol A (Fig. 2). Additionally, we showed that bisphenol A noncompetitively inhibited the aminopyrine $N$-demethyalse activity by CYP2C8 and that bisphenol A exhibited a mixed-type inhibition for $S$-mephenytoin 4-hydroxylation by CYP2C19 (Figs. 3 and 4). Knaak and Sullivan ${ }^{18}$ ) reported that bisphenol A was metabolized to hydroxylated bisphenol A in rats; it has also been suggested that the hydroxylated bisphenol $\mathrm{A}$ is further oxidized to bisphenol- $O$ quinone through bisphenol semiquinone in rats and that this semiquinone and/or quinone is the ultimate DNA binding metabolites of bisphenol A. ${ }^{6}$ Additionally, the formation of adducts in DNA by bisphenol $\mathrm{A}$ in the presence of microsomal activation system has been shown to be markedly decreased by CYP inhibitors, ${ }^{7)}$ suggesting that the metabolism of bisphenol A to the ultimate DNA binding metabolites is mediated by hepatic CYPs. Therefore, one possible explanation of our observations is that the biotransformation from bisphenol A to bisphenol semiquinone and/or bisphenol- $O$ quinone by human hepatic CYPs plays an important role in
(A)

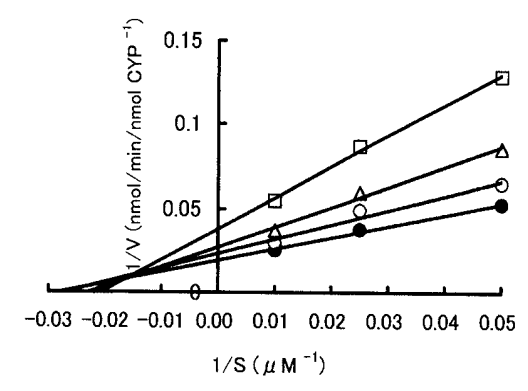

(B)

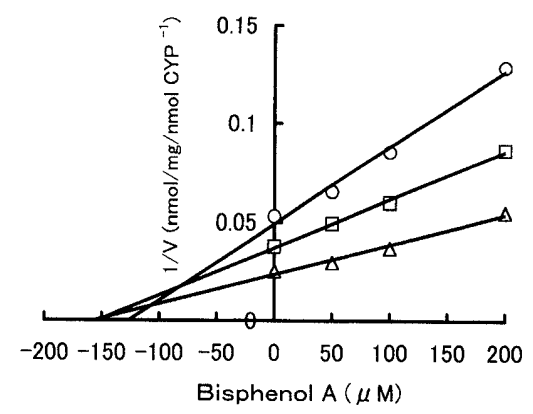

Fig. 4. Inhibitory Effect of Bisphenol A on S-Mephenytoin 4-Hydroxylation by CYP2C19

(A) Lineweaver-Burk presentation of the inhibitory effect of bisphenol A on $S$ mephenytoin 4-hydroxylation by CYP2C19. CYP2C19 was incubated for 5 min with 1 mM NADPH and S-mephenytoin concentrations ranging from 10 to $100 \mu \mathrm{M}$ in the absence $(\bullet)$ or the presence of $50 \mu \mathrm{M}(O), 100 \mu \mathrm{M}(\triangle)$, or $200 \mu \mathrm{M}(\square)$ bisphenol A. (B) Dixon plot illustrating the $S$-mephenytoin-bisphenol A interaction. The concentrations of $S$-mephenytoin were $10 \mu \mathrm{M}(\bigcirc), 20 \mu \mathrm{M}(\square)$, and $40 \mu \mathrm{M}(\triangle)$.

the inhibition of the CYP-mediated reactions.

Bisphenol A inhibited the aminopyrine $N$-demethylation by human hepatic CYPs, especially CYP2C8 and the CYP2C19 mediated $S$-mephenytoin 4-hydroxylation. The inhibition constants ( $K_{\mathrm{i}}$ values) of bisphenol A on aminoipyrine $N$-demethylase activity by CYP2C8 and $S$-mephenytoin 4-hydroxylase activity by CYP2C19 were $97 \mu \mathrm{M}$ and 113 $\mu \mathrm{M}$, respectively. The European Union Commission has established a tolerance migration limit in food of $3 \mathrm{mg} / \mathrm{kg}$ for bisphenol A. ${ }^{19)}$ Biles et al. ${ }^{19)}$ reported that the levels of bisphenol A from epoxy-coated can surfaces to infant formula concentrates ranged from 0.1 to $13 \mathrm{ppb}(57 \mathrm{~nm})$, and Brotons et al. ${ }^{20)}$ extracted bisphenol A from the liquid of canned food ranged from 0 to $33 \mu \mathrm{g}$ per can. When the substrate concentration is much lower than $K_{\mathrm{m}}$ value $\left(\mathrm{S} \ll K_{\mathrm{m}}\right)$, the degree of inhibition $(\mathrm{R})$ can be expressed by the following equation, independent of the inhibition type, except in the case of uncompetitive inhibition, ${ }^{21,22)}$

$$
\mathrm{R}=1 /\left(1+\mathrm{Iu} / K_{\mathrm{j}}\right)
$$

where Iu is the unbound concentration of the inhibitor. Additionally, when the absorption rate is maximum, the maximum influx rate of the inhibitor into liver $\left(V_{\text {in,max }}\right)$ can be expressed as,

$$
V_{\text {in,max }}=\mathrm{I}_{\text {max }}+\left(\mathrm{k}_{\mathrm{a}} \cdot \mathrm{D} / \mathrm{Q}_{\mathrm{H}}\right) \cdot \mathrm{F}_{\mathrm{a}}
$$

where $I_{\max }, k_{a}, D, Q_{H}$ and $F_{a}$ represent the maximum plasma concentration of the inhibitor in the circulation, absorption rate constant, dose, and hepatic blood flow, and the fraction absorbed from the gastrointestinal tract into the portal vein, 
respectively. However, there are few reports on the plasma concentrations of bisphenol A after dosing and the plasma protein binding. To further evaluate potential hazards posed by bisphenol A to human, more information is needed on its exposure and pharmacokinetics.

In summary, we showed that bisphenol $\mathrm{A}$ inhibited the aminopyrine $N$-demethyalse activity mediated by several human hepatic CYPs, especially CYP2C8, and that bisphenol A exhibited a mixed-type inhibition for $S$-mephenytoin 4hydroxylation by CYP2C19.

\section{REFERENCES AND NOTES}

1) Present address: Biopharmaceutical and Pharmacokinetic Research Laboratories, Fujisawa Pharmaceutical Co., Ltd., Kashima, Yodogawa-ku, Osaka 532-8514, Japan.

2) Krishman A. V., Stathis P., Permush S. F., Tokes L., Feldman D., Endocrinology, 132, 2279-2286 (1993).

3) Ashby J., Tennant R.W., Mutat. Res., 204, 17-115 (1988).

4) Guengerich F. P., FASEB J., 6, 745-748 (1992).

5) Gonzalez F. J., Pharmacol. Ther., 45, 1-38 (1990).

6) Atkinbson A., Roy D., Biochem. Biophys. Res. Commun., 210, 424433 (1995).
7) Atkinbson A., Roy D., Eenviron. Mol. Mutagen., 26, 60-66 (1995).

8) Hanioka N., Jinno H., Nishimura T., Ando M., Arch. Toxicol., 72, 387-394 (1998).

9) Guengerich F. P., J. Pharmacokin. Biopharm., 24, 521-533 (1996).

10) Aoyama T., Korzekwa K., Nagata K., Gillette J., Gelboin H. V., Gonzales F. J., Endocrinology, 126, 3101-3106 (1990).

11) Imaoka S., Terano Y., Funae Y., Biochem. Pharmacol., 105, $1041-$ 1050 (1996)

12) Niwa T., Yabusaki Y., Homma K., Matsuo N., Tatsuta K., Ishibashi F., Katagiri M., Xenobiotica, 28, 539-547 (1998).

13) Niwa T., Sato R., Yabusaki Y., Ishibashi F., Katagiri M., Xenobiotica, 29,187-193 (1999).

14) Omura T., Sato R., J. Biol. Chem., 239, 2379-2385 (1964).

15) Nash T., Biochem. J., 55, 416-421 (1953).

16) Dixon M., Biochem. J., 55, 170-171 (1953).

17) Clarke S. E., Xenobiotica, 28, 1167-1202 (1998).

18) Knaak J. B., Sullivan L. J., Toxicol. Appl. Pharmacol., 8, 175-184 (1966).

19) Biles J. E., McNeal T. P., Begley T. H., J. Agric. Food Chem., 45, $4697-4700$ (1997).

20) Brotons J. A., Olea-Serrano M. F., Villaobos M., Pedraza V., Olea N., Environ. Health Perspect., 103, 608-612 (1995).

21) Ito K., Iwatsubo T., Kanamitsu S., Nakajima Y., Sugiyama Y., Ann. Rev. Pharmacol. Toxicol, 38, 461-499 (1998).

22) Ito K., Iwatsubo T., Kanamitsu S., Ueda K., Suzuki H., Sugiyama Y., Pharmacol. Rev., 50, 387-411 (1998). 